\title{
Cortical Networks Produce Three Distinct 7-12 Hz Rhythms during Single Sensory Responses in the Awake Rat
}

\author{
Adriano B. L. Tort, ${ }^{1,2}$ Alfredo Fontanini, ${ }^{3}$ Mark A. Kramer, ${ }^{4}$ Lauren M. Jones-Lush, ${ }^{5}$ Nancy J. Kopell, ${ }^{4 \star}$ \\ and Donald B. Katz ${ }^{6 *}$ \\ ${ }^{1}$ Edmond and Lily Safra International Institute of Neuroscience of Natal and ${ }^{2}$ Federal University of Rio Grande do Norte, Natal, RN 59066, Brazil, \\ ${ }^{3}$ Department of Neurobiology and Behavior, State University of New York at Stony Brook, Stony Brook, New York 11794, ${ }^{4}$ Department of Mathematics and \\ Statistics, Boston University, Boston, Massachusetts 02215, ${ }^{5}$ Department of Physical Therapy and Rehabilitation Science/Department of Anatomy and \\ Neurobiology, University of Maryland School of Medicine, Baltimore, Maryland 21201, and 'Department of Psychology/Program of Neuroscience/Volen \\ National Center for Complex Systems, Brandeis University, Waltham, Massachusetts 02454
}

Cortical rhythms in the $\alpha / \mu$ frequency range (7-12 Hz) have been variously related to "idling," anticipation, seizure, and short-term or working memory. This overabundance of interpretations suggests that sensory cortex may be able to produce more than one (and even more than two) distinct $\alpha / \mu$ rhythms. Here we describe simultaneous local field potential and single-neuron recordings made from primary sensory (gustatory) cortex of awake rats and reveal three distinct 7-12 Hz de novo network rhythms within single sessions: an "early," taste-induced $\sim 11 \mathrm{~Hz}$ rhythm, the first peak of which was a short-latency gustatory evoked potential; a "late," significantly lower-frequency $(\sim 7 \mathrm{~Hz})$ rhythm that replaced this first rhythm at $\sim 750-850 \mathrm{~ms}$ after stimulus onset (consistently timed with a previously described shift in taste temporal codes); and a "spontaneous" spike-and-wave rhythm of intermediate peak frequency ( $\sim 9$ $\mathrm{Hz}$ ) that appeared late in the session, as part of a oft-described reduction in arousal/attention. These rhythms proved dissociable on many grounds: in addition to having different peak frequencies, amplitudes, and shapes and appearing at different time points (although often within single $3 \mathrm{~s}$ snippets of activity), the early and late rhythms proved to have completely uncorrelated session-to-session variability, and the spontaneous rhythm affected the early rhythm only (having no impact on the late rhythm). Analysis of spike-to-wave coupling suggested that the early and late rhythms are a unified part of discriminative taste process: the identity of phase-coupled single-neuron ensembles differed from taste to taste, and coupling typically lasted across the change in frequency. These data reveal that even rhythms confined to a narrow frequency band may still have distinct properties.

\section{Introduction}

The organized activity of neural networks, as reflected in multi-neuronal, local field potential (LFP), electroencephalographic, or magnetoencephalographic recordings, frequently presents a rhythmic quality. Oscillatory activity between 7 and 12 $\mathrm{Hz}$, for instance, has been prominently observed during sensory cortical recordings. This rhythm is variously known as $\alpha$ and $\mu$. Rhythmicity in the $\alpha / \mu$ range dominates sensory cortical (and thalamic) recordings in a variety of awake animal preparations, but this ease of detection has not been matched with an ease of interpretation. Depending on the researcher and context, $\alpha / \mu$ rhythmicity has been

Received Dec. 7, 2009; revised Jan. 20, 2010; accepted Feb. 15, 2010.

This work was supported by the Burroughs Wellcome Fund (A.B.L.T., N.J.K.), the National Science Foundation (N.J.K.), Fundação de Apoio à Pesquisa do Estado do Rio Grande de Norte and Conselho Nacional de Desenvolvimento Científico e Tecnológico, Brazil (A.B.L.T.), National Institute on Deafness and Other Communication Disorders Grant R03-DC-008885 (A.F.), and National Institutes of Health Grant R01-DC-007102 (D.B.K.). We are grateful to members of the Katz laboratory and members of the Cognitive Rhythms Collaborative for helpful discussions.

*N.J.K. and D.B.K. contributed equally to this work.

Correspondence should be addressed to either of the following: Adriano B. L. Tort, Edmond and Lily Safra International, Institute of Neuroscience of Natal, Rua Prof. Francisco Luciano de Oliveira, 2460, Natal, RN 59066, Brazil, E-mail: tort@natalneuro.org.br; or Donald B. Katz, Department of Psychology, MS 062, Brandeis University, 415 South Street, Waltham, MA 02454-9110, E-mail: dbkatz@brandeis.edu.

DOI:10.1523/JNEUROSCI.6051-09.2010

Copyright $\odot 2010$ the authors $\quad 0270-6474 / 10 / 304315-10 \$ 15.00 / 0$ suggested to indicate neural idling (i.e., relaxed resting) (Tiihonen et al., 1989; Pfurtscheller et al., 1997; Fontanini and Katz, 2005), absence seizure (Shaw, 2007), task "preparation" (Nikouline et al., 2000; Fanselow et al., 2001), and memory function (Sauseng et al., 2002; Palva and Palva, 2007; Freunberger et al., 2008). At this time, there is little consensus as to the ultimate role played by $\alpha / \mu$ rhythms in sensory function.

Given this proliferation of interpretations, it is worth asking whether the various researchers involved in the debate are actually observing the same rhythms. Recent data has suggested that several of the previously defined frequency ranges $-\theta(4-7 \mathrm{~Hz})$ and $\gamma$ $(30-80 \mathrm{~Hz})$, for instance-actually conflate distinct subranges that differ in either frequency or spatial source, as well as in function (Rojas-Líbano and Kay, 2008; Tort et al., 2008; van der Meer and Redish, 2009), and that $\alpha$ generators in different cortical regions may be dissociable (Bollimunta et al., 2008). The $7-12 \mathrm{~Hz}$ range may also be divisible into multiple components: different patterns of singlesite and across-brain coherence can be observed depending on the specifics of the sensory and memory tasks being performed (Klimesch, 1999). Notably, however, it is seldom suggested that multiple rhythmic peaks between 7 and $12 \mathrm{~Hz}$ can be discerned (but see Duffy et al., 1984; Könönen and Partanen, 1993).

We have shown previously that time-extensive neural responses to taste stimuli break down into temporal "epochs," with identity 
coding giving way to palatability coding at $\sim 800 \mathrm{~ms}$ after stimulus delivery (Katz et al., 2001a; Fontanini and Katz, 2006; Jones et al., 2007; Grossman et al., 2008). Here, we show that phasic taste delivery to the tongue induces protracted $7-12 \mathrm{~Hz}$ activity in gustatory cortex (GC) and demonstrate that the early and late parts of the GC taste responses are associated with distinct LFP rhythms; furthermore, when attentive coding gives way to general (i.e., not taste-specific) "disengagement" after $\sim 1$ h of recording, a third rhythm appears, again in the $7-12 \mathrm{~Hz}$ band (Fontanini and Katz, 2005) (for examples of this inattentive rhythm from other sensory cortices, see Bezdudnaya et al., 2006; Shaw, 2007). We go on to relate taste-responsive single-neuron activity to the underlying rhythms, demonstrating that the two stimulus-induced rhythms, although distinguishable from each other and the third 7-12 Hz rhythm, appear to be a unified part of cortical taste processing.

\section{Materials and Methods}

\section{Subjects}

Female Long-Evans rats (250-300 g at the time of surgery) were maintained on a $12 \mathrm{~h}$ light/dark schedule, with experiments performed in the light portion of the cycle. Unless otherwise specified, chow and water were available ad libitum.

\section{Surgery}

Anesthesia was induced and maintained using an intraperitoneal injection of a ketamine, xylazine, and acepromazine mixture (100, 5, and 1 $\mathrm{mg} / \mathrm{kg}$, respectively, for induction; $20-30 \%$ of induction dose for maintenance). Once anesthetized, rats were placed on a stereotaxic frame, at which time holes were bored in the skull for ground screws and electrode bundles. Two microelectrode assemblies were lowered to $0.6 \mathrm{~mm}$ above layer 5 of GC bilaterally, guided by stereotaxic measurements (anteroposterior, $1.4 \mathrm{~mm}$; mediolateral, $\pm 5 \mathrm{~mm}$ from bregma; dorsoventral, $-4.0 \mathrm{~mm}$ from dura). Once in position, each electrode bundle was cemented to the skull with dental acrylic, as was a bolt for restraining head movements. After electrode implantation, intraoral cannulae (IOC) were inserted bilaterally (Phillips and Norgren, 1970; Fontanini and Katz, 2005). The scalp was then sutured, and antibiotic ointment was applied to the wound. The rat received a subcutaneous injection of penicillin $(0.1 \mathrm{cc})$ immediately after surgery and again $2 \mathrm{~d}$ later.

\section{Training}

Starting $2 \mathrm{~d}$ after surgery, the electrodes were lowered $\sim 70-140 \mu \mathrm{m}$ every second or third day, until they were buried in layer 5 of the tasteresponsive part of insular cortex (Katz et al., 2001a, 2002). Seven days after surgery, rats were begun on a regimen of mild water restriction (30 $\min \mathrm{H}_{2} \mathrm{O}$ per day). After $3 \mathrm{~d}$ of water restriction, they were introduced to the experimental head-restraint chamber (Welsh et al., 1995; Bermejo et al., 1998; Katz et al., 2001a) and progressively trained to press a lever for water delivery at longer and longer delays aided by a $5 \mathrm{kHz}$ tone that signaled taste availability. Within 3-10 sessions, rats were effectively able to sit quietly through a $40 \mathrm{~s}$ period between lever presses and to receive an experimenter-administered water delivery $20 \mathrm{~s}$ after each self-administration (for details, see Fontanini and Katz, 2005, 2006). At this point, randomly selected tastes were substituted for both experimenter- and self-administered water aliquots, and recording sessions commenced. Thus, each animal provided data for self- and experimenter-administered taste responses (only the latter are analyzed here) in each session, with trials interleaved.

Sessions occurred during late afternoon; rats received water after training, and the electrodes were lowered by $\sim 180 \mu \mathrm{m}$. Each of three rats participated in four taste/recording sessions, with distinct placements and neural isolations each time.

\section{Taste delivery}

Tastes were delivered, under nitrogen pressure through a set of four polyimide tubes inserted into one IOC, in $40 \mathrm{~ms}$ squirts during periods of quiet waiting by the rat (interval between delivery longer than $\sim 20 \mathrm{~s}$ ). Tastes included $35 \pm 5 \mu$ l of $100 \mathrm{~mm} \mathrm{NaCl}, 100 \mathrm{~mm}$ sucrose, $100 \mathrm{~mm}$ citric acid, and $1 \mathrm{~mm}$ quinine $\mathrm{HCl}$. These are the prototypical salty, sweet, sour, and bitter tastes, respectively (Norgren, 1990; Smith and St John, 1999); the former two are palatable to rats, and the latter two are aversive. Every delivery of a taste was followed, after $5 \mathrm{~s}$, by a water rinse delivered through a second IOC. To maximize the number of taste trials delivered, water was only delivered as a rinse; given that water functions as a taste, by virtue of reducing salt concentrations and activating taste-related somatosensory receptors on the tongue (Bartoshuk et al., 1964; Katz et al., 2001a; Verhagen, 2007), it is likely that water results would be similar.

\section{Electrophysiology}

Each electrode bundle included $1625 \mu \mathrm{m}$ wires (nichrome microwires insulated with Formvar, impedance of $\sim 3-500 \mathrm{k} \Omega$ ) glued to a small microdrive (Katz et al., 2001b). Recordings from all microwires, made during each session that included tastes, were amplified, bandpass filtered at 300-8000 $\mathrm{Hz}$, and digitized (Plexon). Sixteen of the raw signals were also split off to a separate amplifier with filtering set for LFP recording (bandpass $0.3-90 \mathrm{~Hz}$ ) and from there to a computer, where they were digitized at $1000 \mathrm{~Hz}$.

Single neurons of $>3: 1$ signal-to-noise ratio were isolated using a waveform template algorithm, augmented with offline cluster cutting software (Plexon) and inspection of interspike interval plots for proper refractory periods. The properties (baseline firing rates, response rates) of units isolated with this method are similar to those of units isolated with more classic (acutely driven high-impedance sharp electrodes) techniques (Katz et al., 2001b). All isolations were reliable for the entire duration of the experiment. A total of 109 layer 5 GC neurons were isolated across 12 sessions with three rats.

\section{Data analysis}

All analyses were done using built-in and custom routines written in MATLAB (MathWorks), on data that had previously been divided into "attentive" and "inattentive" periods on the basis of multiple criteria (for details, see Fontanini and Katz, 2005, 2006).

Power spectral density estimation. For the power spectra estimation, each data segment was first multiplied by a Hamming window and then transformed to the frequency domain by a fast Fourier transform (FFT). The absolute value of the transformed data defined the power spectrum. For the purposes of this paper, we refer to an LFP as "rhythmic" when a significant peak in power could be observed in a restricted frequency range in the power spectra. This does not imply that the LFP during these periods contained only oscillatory components, nor that the frequency of response was noiseless. Typically, however, oscillatory properties could be clearly observed in raw signals deemed rhythmic (see Fig. 1).

In a set of control analyses, we confirmed the statistical significance of the spectral peaks located in the $7-12 \mathrm{~Hz}$ range. We first approximated the noise level by fitting a $1 / f$ curve to the power spectral density for frequencies $>70 \mathrm{~Hz}$. We then extended this curve to lower frequencies and verified that the power spectral density confidence intervals in the 7-12 Hz range (obtained by means of a jackknife procedure available in the Chronux toolbox; http://www.chronux.org/) did not include this curve (for details on this procedure, see Shumway and Stoffer, 2005).

Time-frequency decomposition. The time-frequency decomposition used 500-ms-long sliding time windows shifted in steps of $50 \mathrm{~ms}$ (i.e., $90 \%$ overlap between windows). This was obtained using the spectrogram function from the MATLAB Signal Processing Toolbox. The $z$-score normalization used the mean and the SD over the power values, at each frequency, obtained for the time windows in the baseline ("pretaste") period.

Filtering. LFP bandpass filtering was obtained using a linear finite impulse response filter by means of the eegfilt function from the EEGLAB toolbox (Delorme and Makeig, 2004), which is available for free download at http://sccn.ucsd.edu/eeglab/. The filter order depends on the lowfrequency cutoff, and it is given by three times the ratio of the sampling frequency to the low-frequency cutoff (rounded to the nearest smaller integer). The eegfilt function calls the MATLAB routine filtfilt, which applies the filter forward and then again backward, to ensure that phase delays introduced by the filter are nullified.

Evoked response. The gustatory evoked potential (GEP) response was obtained using the erpimage routine from the EEGLAB toolbox (Delorme and Makeig, 2004), which averages the LFPs over trials. How- 


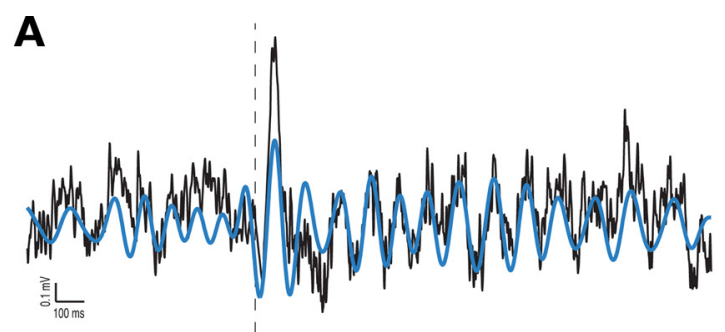

C

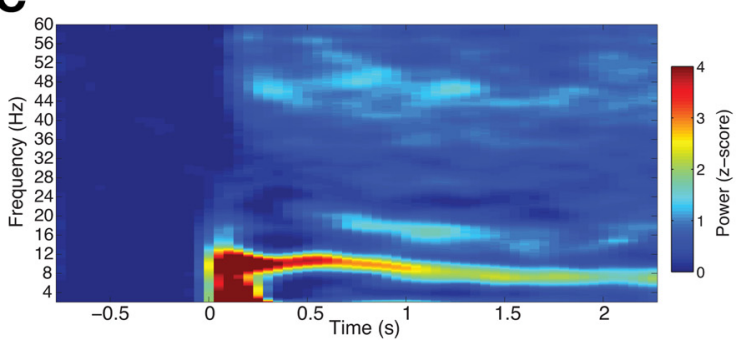

B

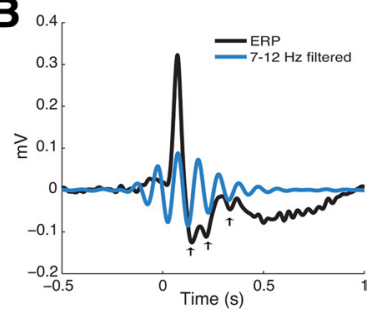

D

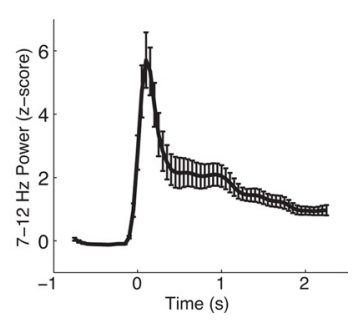

Figure 1. GC activity evoked by taste stimulation. $\boldsymbol{A}$, Taste administration (vertical dashed line) induces a GEP, followed by 7-12 $\mathrm{Hz}$ oscillations in a GCLFP. A version of the same signal, bandpass filtered between 7 and $12 \mathrm{~Hz}$, is overlain in blue, showing that the GEP appears to be the first peak of the taste-induced rhythmic activity. $\boldsymbol{B}, \mathrm{A}$ whole-dataset (i.e., across-rat, across-session, across-electrode, across-taste, across-trial) average LFP, showing the reliability of both the GEP and the first few 7-12 Hz cycles (troughs noted with arrows). Again, the bandpass filtered version is overlain in blue. $\boldsymbol{C}$, A spectrogram of the GC taste response, showing a broad (2-12 Hz) GEP lasting 2-300 ms, followed by a narrow-band oscillatory response that is visible for at least $2 \mathrm{~s}$. D, Seven to $12 \mathrm{~Hz}$ power rises quickly with taste delivery and then decreases but stays significantly above baseline.

ever, for the correlation between the GEP peaks and the 7-12 Hz peak times, which is an analysis that requires the assessment of the GEP within single trials, the GEP peak time in each trial was estimated after removing fast components that disappear in the averaged response; this was obtained by low-pass filtering the raw signal below $30 \mathrm{~Hz}$.

Phase extraction. The $7-12 \mathrm{~Hz}$ phase time series $(\varphi)$ was computed from the Hilbert transform of the 7-12 Hz filtered signal, which was obtained using the hilbert routine from the MATLAB Signal Processing Toolbox. The information about instantaneous phase was used to assess intratrial and intertrial phase coherence measures (subsection below) as well as to compute the spike phase histograms.

Phase consistency and intertrial coherence. The phase coherence levels among 7-12 Hz time series $\Phi(t)=\left[\varphi_{1}(t), \ldots, \varphi_{\mathrm{N}}(t)\right]$ can be estimated at each time $t$ by the following formula: $\operatorname{abs}(\operatorname{mean}(\exp (i \Phi(t))))$, where the mean is taken over all unitary vectors $\exp \left(i \varphi_{j}(t)\right), j=1 \ldots N(N$ is the number of trials), and abs computes the length of this mean vector. This equation produces a number on $[0,1]$, with 0 denoting no phase locking and 1 perfect phase locking among the phase time series (notice that 1 is obtained when all phases $\varphi_{j}(t)$ have the same value across trials, which gives rise to a mean vector of length 1 ). This measure has been named in different ways by different authors (Bruns, 2004). When the $\varphi_{j}$ time series denotes the phase difference between two electrodes in the same trial, this measure is often called "phase consistency" (PC). When $\varphi_{j}$ values are obtained from the same electrode in different trials, this measure has been called as event-related "intertrial coherence" (ITC) or also "phasereset index." We followed the PC and ITC name conventions in the present work. For the comparison of the time course of the changes in ITC and PC, we have normalized this measure by subtracting the minimum value and dividing the result by the difference between the maximum and minimum values [i.e., $\mathrm{ITC}_{\text {norm }}=\left(\mathrm{ITC}-\mathrm{ITC}_{\min }\right) /\left(\mathrm{ITC}_{\max }-\mathrm{ITC}_{\min }\right)$, and similarly for PC].

Power spectra of single neurons. The power spectral density of GC neuron spike trains was estimated using rate histograms. The bin size for the rate histograms were computed using the following formula: bin $=1 . /\left(2{ }^{*}\right.$ maximum_frequency), with maximum frequency set to $20 \mathrm{~Hz}$. Once determined, the rate histograms were detrended (i.e., the linear regression was subtracted), a Hann window was applied, and an FFT was computed on the result.

Correlation studies. Linear correlations were assessed by means of the Pearson's product moment coefficient $(r)$. A $p<0.05$ in transformed data denoted a statistically significant correlation. For the correlation of peak frequencies, a normalization was used to control for between-animal differences; this was obtained by dividing each value by the mean value in a given rat.

\section{Results}

\section{Taste-induced 7-12 Hz rhythm}

Analog signals from chronically indwelling drivable microwires, 16 in each GC, were each split into pairs of independently filtered channels, enabling us to simultaneously sample LFP and single-neuron taste responses (sweet, salty, sour, and bitter) in awake rats. Initial analyses were solely taken from the first hour of each session, during which time the animal was attentive (Fontanini and Katz, 2005, 2006) (see below for analyses including inattentive processing). Figure $1 A$ shows a representative LFP trace, this one induced by the administration of $\mathrm{NaCl}$ through the intraoral cannula; this trial was representative of responses to all tastes. A large deflection of the LFP followed closely on the heels of taste delivery (time noted with a vertical dotted line), after which the signal could be observed by eye to become notably more rhythmic. A filtered version of the same trace $(7-12 \mathrm{~Hz}$ bandpass, dark blue curve), overlain on the wide-band signal, demonstrates that an increase in the $7-12 \mathrm{~Hz}$ rhythm characterizes the taste response; taste administration significantly increases the similarity between the overall signal and its $7-12 \mathrm{~Hz}$ component (average $r=0.42 \pm 0.001$ ) compared with the period preceding the stimulus (average $r=0.31 \pm 0.0015, t$ test comparing the correlations, $p<0.01$ ).

Figure $1 B$ shows the grand response average across all rats (three), sessions (four per rat), tastes (four per session), trials $(\sim 10$ per taste), and electrodes (eight per GC), with the average of filtered traces again overlain [the apparent prestimulus rhythmicity is an artifact of filtering (supplemental Fig. 1, available at www.jneurosci.org as supplemental material)], such that the reliability of the first deflection (a GEP) and timing of the first several cycles (arrows) can be observed (results are similar for single rats and sessions). The GEP, which is higher and a bit broader than subsequent peaks, elevates power at all frequencies below $15 \mathrm{~Hz}$ during the first 2-300 ms of the response (Fig. 1C). Although the excess $7-12 \mathrm{~Hz}$ power during this first specific time period could be described as spillover from lower frequencies, power remains elevated above baseline in the $7-12 \mathrm{~Hz}$ range for at least $2 \mathrm{~s}$ after subsidence of the GEP, such that a tight and significant peak, elevated in power over surrounding frequencies, appears (Fig. $1 D$ ). Subtle elevations in $14-20 \mathrm{~Hz}$ power can also be observed in Figure 1, $B$ and $C$, as can patchy increases in 30-60 $\mathrm{Hz}$ power; these phenomena may be worth studying in their own right, but, for the remainder of Results, we will focus on the much larger modulations of 7-12 $\mathrm{Hz}$ power.

The GEP may represent the activation of GC synapses via transmission of somatosensory information rather than reflecting gustatory processing per se. The latency of this potential (time-to-peak $<100 \mathrm{~ms}$ ) matches that of somatosensory content in GC single neurons and precedes that of chemosensory content by $>50 \mathrm{~ms}$ (Katz et al., 2001a); furthermore, neither this latency, 
A

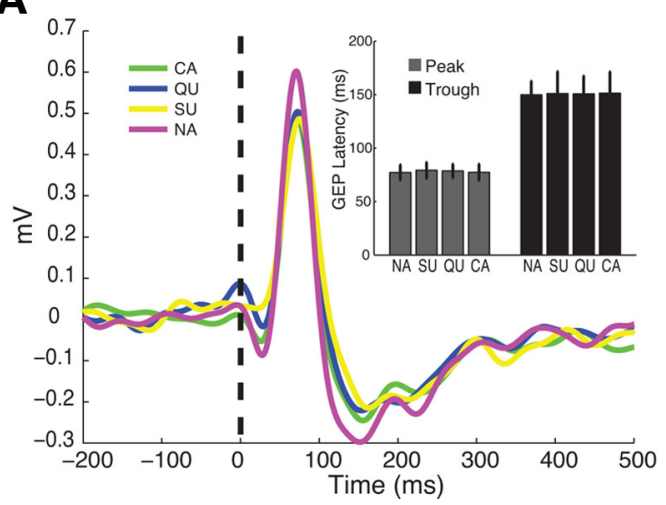

B

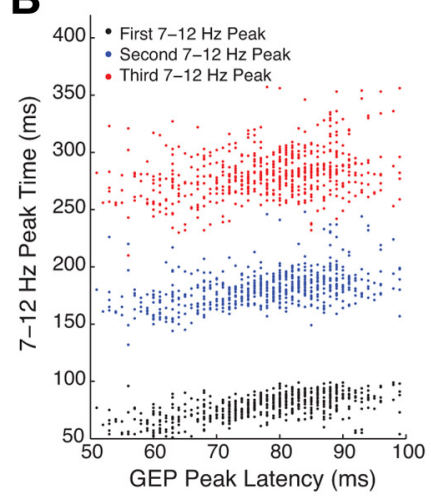

C

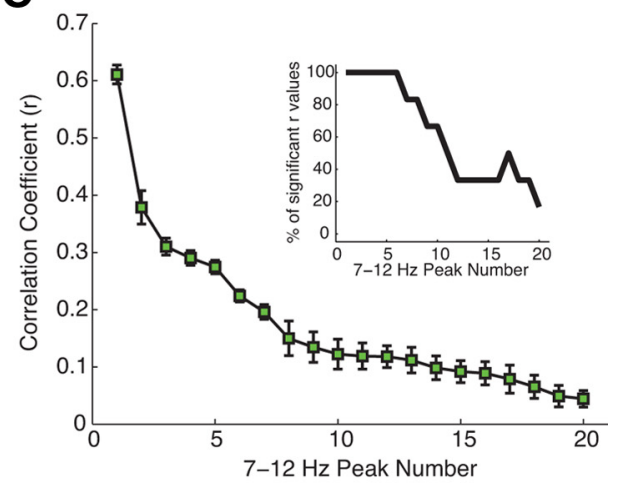

Figure 2. The gustatory evoked potential begins oscillatory processing. $A$, The averaged GEPs for each administered taste (NA, sodium chloride; SU sucrose; $Q U$, quinine; $C A$, citric acid) look remarkably similar to each other (the NA GEP looks slightly larger than that for the other tastes, but this difference is not significant). Time is on the $x$-axis, with taste delivery at 0; voltage (millivolts) is on they-axis. The inset shows that the latencies of GEP peaks and troughs ( $y$-axis) were also similar for all tastes. $\boldsymbol{B}$, For the data from this representative GC, the peak latency of the GEP was highly correlated with the peak latencies of the first, second, and third peaks of the signal bandpass filtered between 7 and $12 \mathrm{~Hz}$, that is, variations in the latency of the GEP predicted variations in the oscillations. Each individual trial contributed three dots, one for each of the 7-12 Hz peaks. $C$, The correlations between GEP peak latency and the first 20 peaks of the oscillation, across the entire dataset. This correlation was significant $(p<0.05)$ in all six GCs for the first six cycles. The likelihood of the GEP-to-peak correlation reaching significance in an individual GC begins to decline after the sixth cycle (inset).

latency to return to baseline, nor amplitude of the GEP shows any evidence of taste specificity (Fig. 2A). That is, there is no chemosensory information in the GEP.

Although (as noted above) the $7-12 \mathrm{~Hz}$ rhythm far outlasts the GEP, the precise timing of the GEP peak is effectively identical to that of the timing of the first $7-12 \mathrm{~Hz}$ peak, pinpointed separately in the filtered signal (Fig. 1A). The GEP peak latency provides a good prediction for the second and third $7-12 \mathrm{~Hz}$ peaks, as well (Fig. $2 B$ shows all such values across all sessions, tastes, and electrodes for one GC in one rat). In fact, across the entire dataset, the correlation between the GEP peak timing and that of the first four rhythmic peaks is $>0.3$ and remains significantly above chance (at $p<0.05$ ) for the first six peaks [Fig. $2 C$, the inset of which shows the proportion of six GCs (two per rat) for which the GEP to $7-12 \mathrm{~Hz}$ peak correlation was significant at that level]. The $7-12 \mathrm{~Hz}$ power is not simply "spillover" of broader-spectrum GEP power, but it does appear to be "kick-started" by the GEP.

\section{Network nature of 7-12 Hz rhythm}

We made simultaneous recordings of eight LFP signals in each hemisphere. Initial populations analyses were restricted to the first hour of each session to avoid late-session changes in attention (Fontanini and Katz, 2006). Stimulus delivery induced a significant increase in between-signal phase coherence $(p<$ $0.05)$ that remained elevated for $>1.0 \mathrm{~s}$ after stimulus delivery, within single trials; this result was nearly identical for within and between hemisphere pairs of signals (Fig. 3A). For contrast, we calculated trial-to-trial 7-12 $\mathrm{Hz}$ phase coherence at single electrodes, an analysis that essentially revealed how reliable the tasteinduced "phase reset" of the $7-12 \mathrm{~Hz}$ oscillation was across individual trials. The fact that between-trial phase coherence immediately soared after stimulus delivery and remained elevated for almost $0.5 \mathrm{~s}$ (Fig. 3A, black trace) demonstrates that taste administration and the subsequent GEP did reset $7-12 \mathrm{~Hz}$ activity to a reliable phase in each trial. The within-trial phase coherence remained elevated for more than twice as long as the between-trial coherence (see inset), however, suggesting that a real-time network mechanism maintained the coherence above and beyond a level that can be explained in terms of a single driving force.

Even if a network mechanism maintained the rhythm, it remained possible that the onset of the taste-induced 7-12 Hz
A

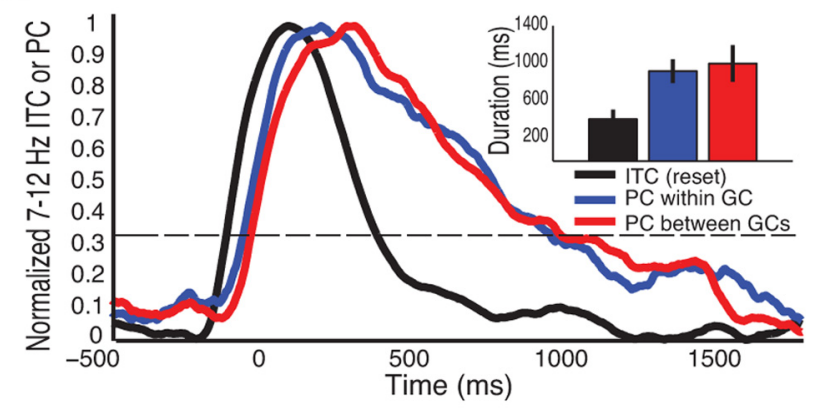

B

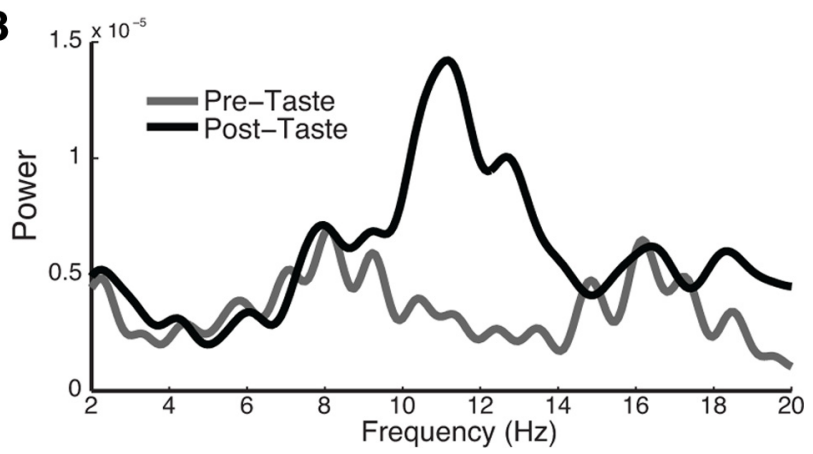

Figure 3. GC taste responses reflect both a phase reset and an increase of oscillatory activity. $A$, Seven to $12 \mathrm{~Hz}$ phase locking between trials was relatively brief compared with within-trial phase locking; that is, the consistency of phase-reset (black trace) returned to baseline levels in $<1 \mathrm{~s}$ (see also Fig. 1B), whereas both within- and between-hemisphere single-trial increases in $7-12 \mathrm{~Hz}$ coherence (blue and red traces, respectively) remained elevated above baseline levels for $\sim 1.5 \mathrm{~s}$. These curves were obtained from the grand average across rats. Inset, The mean duration of phase reset (black bar) - the amount of time in which across-trial phase coherence exceeded one-third of its normalized peak (dashed line) — was less than half of the duration of elevated single-trial response coherence, either within one cortex (blue bar) or between cortices (red bar). B, Spike trains in GC typically showed no excess $7-12 \mathrm{~Hz}$ power in the $1 \mathrm{~s}$ before stimulus delivery (gray trace); in the $1 \mathrm{~s}$ after stimulus delivery, however, $7-12 \mathrm{~Hz}$ power is obvious and significant (black trace).

power could reflect (1) de novo appearance of rhythmic neural activity, (2) a phase reset of neural activity that was already rhythmic but non-phase locked, or (3) both of the above. Although we cannot definitively determine which of these possibilities is correct using extracellular recordings (because such recordings can- 


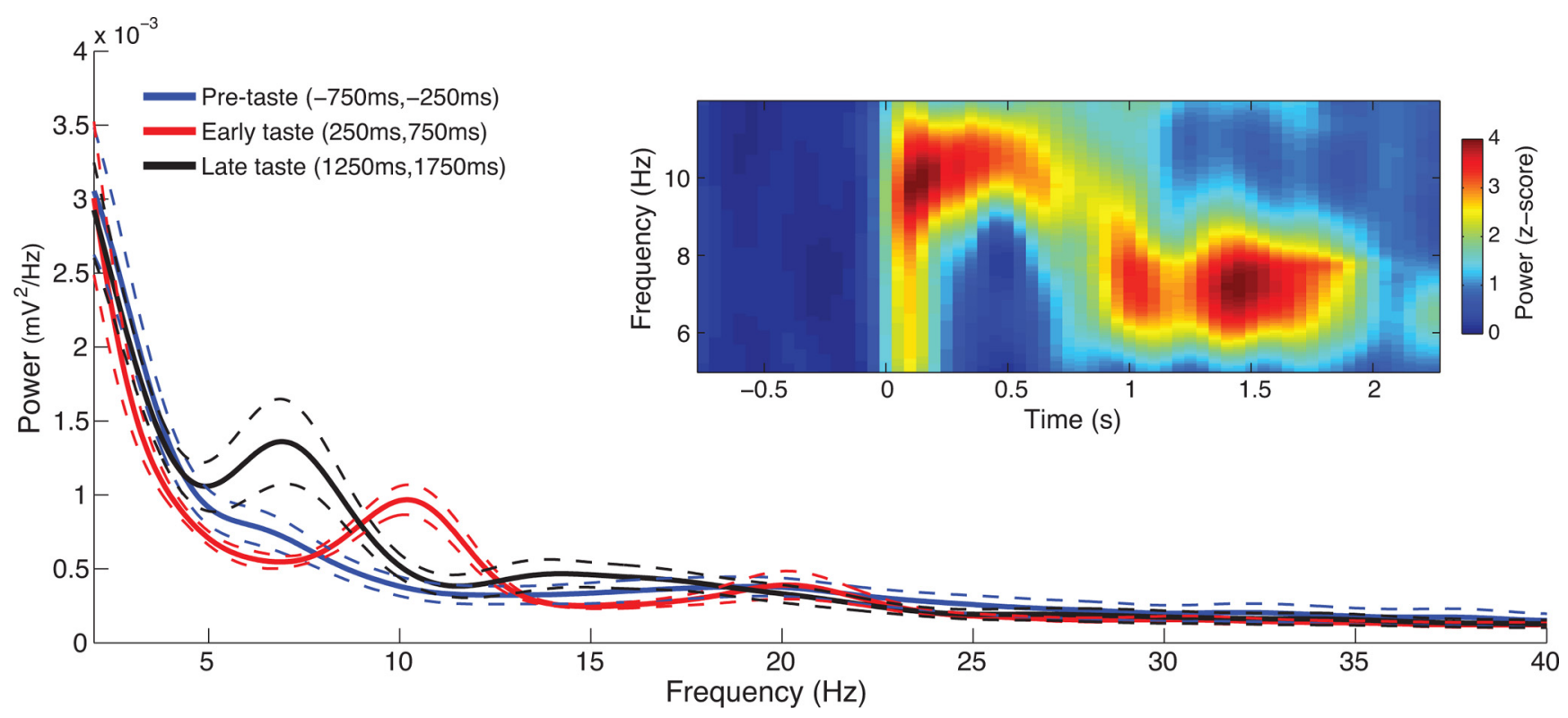

Figure 4. Taste processing actually involves two separate but similar rhythms. The primary frequencies of the early and late taste rhythms (red and black lines, respectively) are distinct from each other and from the spectral content of spontaneous activity (blue line). Dashed lines show the confidence intervals around each spectral analysis. $n=6$ (GCs). The inset shows an example power $\times$ time plot taken from one $\mathrm{GC}$, which clearly reveals the relatively sudden switch in frequencies at $\sim 800 \mathrm{~ms}$ after stimulus delivery.

not rule out the presence of oscillatory membrane potentials), our data strongly suggest that taste administration induces rhythmic LFP properties de novo. Figure $3 B$ shows the power spectrum of the $1 \mathrm{~s}$ prestimulus period (gray trace) and $1 \mathrm{~s}$ poststimulus period (black trace) for an example GC single-neuron spike train. Significant 7-12 Hz power appears only after stimulus onset; in fact, there is essentially no "excess power" in the 7-12 Hz range during spontaneous activity for this (typical) GC neuron (again, during attentive processing; see below) (Fontanini and Katz, 2005), but after taste administration, the power increases significantly. When the entire sample of single neurons $(n=109)$ is examined as a group, $7-12 \mathrm{~Hz}$ power is absent from prestimulus records and is significant after stimulus delivery ( $t$ test of $7-12 \mathrm{~Hz}$ vs other frequencies, $p<0.002)$. This demonstrates that tasteinduced LFP rhythmicity is primarily not the result of phase locking between the already rhythmic (but random phase) activities of single neurons; it is de novo oscillations started at a consistent phase by the GEP and sustained by network mechanisms.

\section{The peak frequency of taste-induced $7-12 \mathrm{~Hz}$ peaks changes suddenly within responses}

A close look at Figure 1 suggests that the $7-12 \mathrm{~Hz}$ rhythmicity that appears during taste administration undergoes a change in peak frequency over the course of $2 \mathrm{~s}$. The inset of Figure $2 C$ also hints at the existence of a break between the first 7 7-12 $\mathrm{Hz}$ cycles, which are significantly correlated with GEP timing, and those that come after. Figure 4 quantifies this apparent change, showing the power spectra and confidence intervals for prestimulus periods (blue), the periods between 250 and $750 \mathrm{~ms}$ after stimulus delivery (red, chosen to avoid inclusion of any spillover from the GEP), and the periods 1250-1750 ms after stimulus delivery (black). It is clear that what we will call the "early taste rhythm" (see Discussion) centered at $10.5 \mathrm{~Hz}$ gets replaced by a "late taste rhythm" centered at $7 \mathrm{~Hz}$. The confidence interval surrounding the early peak does not overlap that of the same frequency band during the later time period (and vice versa); that is, there was no evidence of a $10.5 \mathrm{~Hz}$ oscillation in second second of the re- sponses and no evidence of a $7 \mathrm{~Hz}$ oscillation in the first second. This fact confirms the difference between the early and late rhythms, which are essentially nonoverlapping.

The inset in Figure 4 shows this effect in the time course of spectral power for one GC in one session. For approximately the first $600 \mathrm{~ms}$, the peak frequency straddles $10 \mathrm{~Hz}$, but, by $1.0 \mathrm{~s}$ after taste delivery, a second rhythm in the vicinity of $7 \mathrm{~Hz}$ has replaced the first. It is probably not coincidental that this relatively sudden shift in network frequency occurs at approximately the same time as the previously described shift in GC taste temporal coding, from coding of stimulus identity to coding of stimulus palatability (Katz et al., 2001a; Fontanini and Katz, 2006; Grossman et al., 2008). In fact, the Figure 4 inset probably underestimates the suddenness of the change: analysis of neural ensemble activity strongly suggests that these shifts occur in $<100 \mathrm{~ms}$ but that the exact time of that shift varies from trial to trial in a range of several hundred milliseconds (Jones et al., 2007). Across-trial averages such as the one presented here thus "smear" the apparent time of change across this range.

Note that, although this frequency shift is well timed to reflect a transition in processing that has been described in both single neuron coding (Katz et al., 2001a) and consumptive behavior (Travers and Norgren, 1986), it is not well explained as a reflection of the behaviors themselves: $7 \mathrm{~Hz}$ licking behavior is generated during the first second of taste processing, when the dominant LFP rhythmicity is centered at $10.5 \mathrm{~Hz}$; in fact, this 7 $\mathrm{Hz}$ behavior disappears, to be replaced by much lower-frequency "gapes" and "lateral tongue protrusions" at approximately the same time that $7 \mathrm{~Hz}$ LFP rhythmicity begins (Travers and Norgren, 1986) (see also Discussion).

\section{A total of three distinct GC 7-12 Hz frequency peaks}

We have reported previously the emergence of what we have called "withdrawal," a sudden reduction of attention or arousal at $\sim 1 \mathrm{~h}$ into a session of stimulus delivery to an awake rat (Fontanini and Katz, 2005, 2006). When we examine this portion of the session, we find the sudden appearance of "spontaneous" spike- 
and-wave rhythms with a peak frequency of $9 \mathrm{~Hz}$ accompanying this withdrawal (Fig. 5). This effect of attentional withdrawal is a general one, observed in other sensory cortices (Bezdudnaya et al., 2006; Shaw, 2007). Here, as in these other areas, this pretaste rhythm is typically quenched by stimulus delivery (Fig. $5 A$ ). Still, the late (Fig. $5 B$ ) and less frequently the early (Fig. $5 C$ ) taste rhythm can still be observed to a reduced degree; these surviving early and late taste rhythms are undetectable in Figure $5 \mathrm{~A}$ only because the spontaneous spike-and-wave rhythm is very large (several times the amplitude of the other rhythms, in fact) (Fig. 6) and thus dominates the dynamic range of the color scale. The pretaste LFPs are notably different in wave shape than either of those induced by taste delivery, being much sharper, larger, and less "sine-like" (Fig. 5D).

Looked at together in grand averages across rat and session (Fig. 6), the differences between the peak frequencies of the three described rhythms-pretaste, early taste, and late taste-are obvious despite the fact that all exist within the $7-12 \mathrm{~Hz}$ range that is so often described as monolithic and the fact that all can be recorded within single sessions with the same electrodes. The pretaste rhythm peaks sharply at $9 \mathrm{~Hz}$ and is observed only after attentional withdrawal (Fontanini and Katz, 2005) and only in the time periods between taste administrations. The early taste rhythm peaks at $10.5 \mathrm{~Hz}$ and is only just barely visible late in the session (after withdrawal). The late taste rhythm peaks at $7 \mathrm{~Hz}$ and is strong from the beginnings to the ends of sessions.

Finally, the pattern of interactionsand of the lack of interactions-among these rhythms underscores their distinctiveness. Early and late taste rhythms, both of which evince between-trial and betweensession variability, might be expected to change together. This turns out not to be true, however: variability in the peak frequency of the late taste rhythm is completely uncorrelated to variability in the peak frequency of the early taste rhythm. The peak frequency of the pretaste rhythm, meanwhile, was highly correlated to that of the early taste rhythm that followed close on its heels (Fig. 7A, top) but not at all to that of the late taste rhythm (Fig. 7A, bottom). We investigated this last result further and found that the peak frequency of the early taste rhythm changed significantly after the rat's withdrawal; that is, when the early taste rhythm followed pretaste rhythm, it was typically centered on a frequency $>0.5 \mathrm{~Hz}$ lower (i.e., closer to that of the pretaste rhythm itself) than earlier in the session. The late taste rhythm, meanwhile, was completely unaffected by the pretaste rhythm (Fig. $7 B$ ). Thus, the three rhythms, all centered in the $7-12 \mathrm{~Hz}$ range, differ in (1) shape, (2) amplitude, (3) peak frequency, (4) time of occurrence, and (5) their interrelationships. bars are too small to be seen.
B
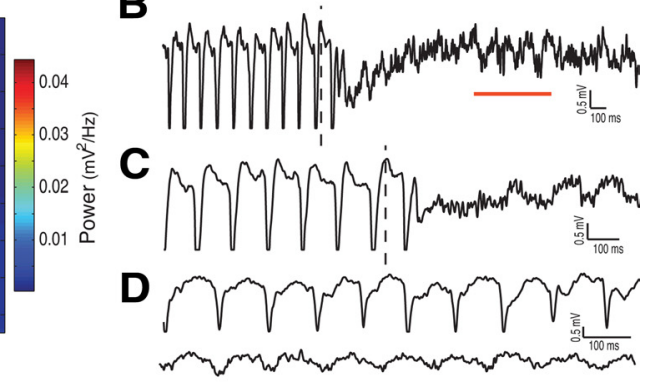

Figure 5. A third distinct rhythm in the same frequency range appears later in the same sessions. $\boldsymbol{A}, \mathrm{An}$ across-trial average becomes rhythmic in the $7-12 \mathrm{~Hz}$ range. At this time, taste delivery (vertical dashed line) primarily inhibits the rhythmicity, owing a case in which the early taste rhythm appears in an inattentive rat (early taste rhythm appeared in $50 \%$ of the inattentive

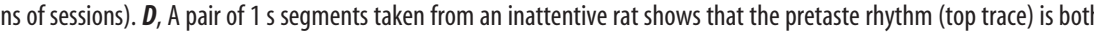
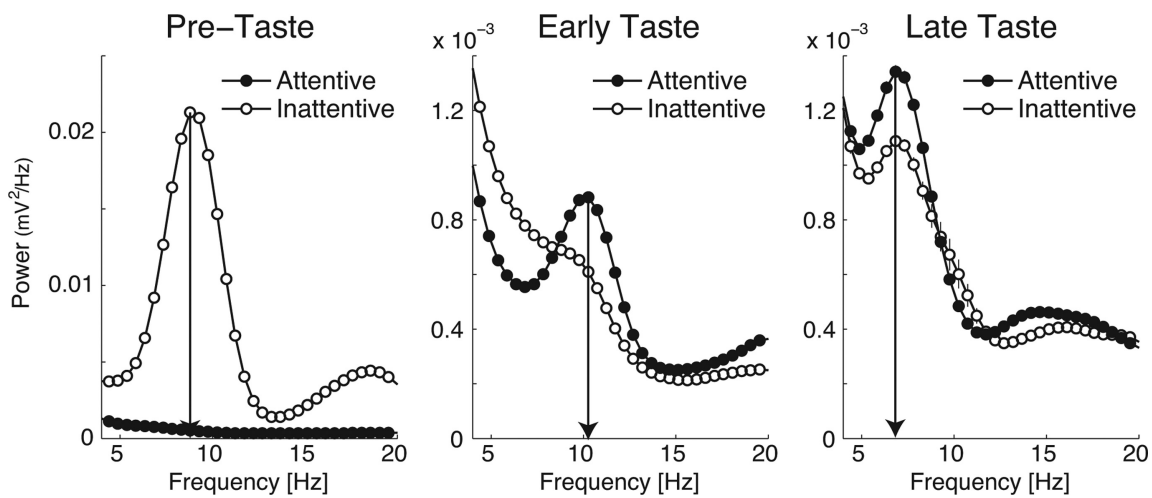

Figure 6. Summary of the different 7-12 Hz rhythms present in GC during a tasting session. The left shows spectral analyses of Katz, 2005, 2006); a strong rhythm centered at $9 \mathrm{~Hz}$ (arrow) appears only in the inattentive rat. The middle analyzes the early taste power centered at $10.5 \mathrm{~Hz}$ is present in the attentive rat but mostly absent in the inattentive rat. The right analyzes the late very different $y$-axis scaling for the left panel, necessitated by the large difference in amplitude between the rhythms. Figure 5 allows direct comparisons of the sizes of the rhythms; here, the focus is on comparing peak frequencies and on comparing attentive and inattentive portions of the sessions for each rhythm. Note as well that every single trace was used for this panel; thus, the error

\section{The early and late taste rhythms are both a part of sensory} processing itself

The significance of the above results depends to some degree on the relevance of the described frequency peaks to sensory processing. The pretaste rhythm is clearly not a specifically tasterelated rhythm; here, we demonstrate that the early and late taste rhythms are in fact involved in taste processing; they are not simply a general index of sensory function.

The most general properties of the rhythms were in fact general to all tastes: the average power in the 7-12 $\mathrm{Hz}$ band was similar for all stimuli ( $\mathrm{NaCl}$, sucrose, quinine, and citric acid) (Fig. 8A); furthermore, the peak frequencies of both rhythms were consistent across taste (Fig. $8 B$ ). This further suggests that the taste rhythms are not motor efference, because rats typically lick more slowly when faced with aversive tastes (Baird et al., 2005).

Taste specificity did emerge in terms of which single GC neurons phase locked with the LFP rhythm. That is, although the LFP itself looked similar from taste to taste, primarily nonoverlapping taste-specific assemblies of neurons were recruited into the rhythm. Figure $8 C$ shows, for a representative single GC neuron, 
A

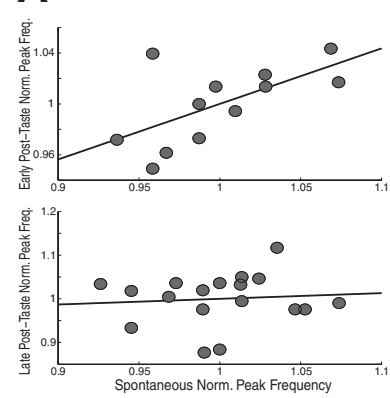

B

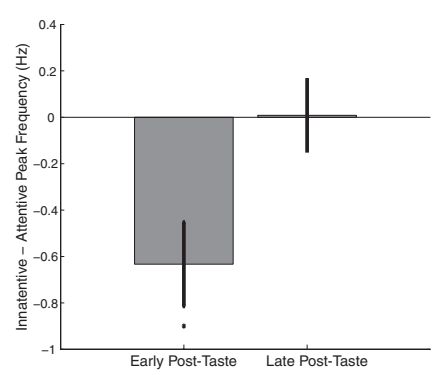

Figure 7. Spontaneous $\sim 9 \mathrm{~Hz} \mathrm{GC}$ rhythm affects early but not late taste processing rhythm. $\boldsymbol{A}$, The top shows that session-to-session variability in the peak frequency of the pretaste rhythm ( $x$-axis) was tightly linked to variability in early taste rhythm ( $y$-axis) during the same part of the session. The solid black line is the best linear fit; the two variables are significantly correlated $(r=0.65, p<0.01$ ). Both variables have been normalized to control for betweenanimal differences in average frequency. The bottom shows that there was no such relationship between the spontaneous and late taste rhythms; the correlation between these two variables was not significant. $\boldsymbol{B}$, The change from attentive to inattentive has no impact on the peak frequency of late taste processing rhythm (right bar) but significantly reduces the peak frequency of early taste processing rhythm (left bar).

the cycle histograms (i.e., the number of taste-responsive action potentials fired during each cycle) for sucrose and quinine and the statistical significance of how well the firing rate modulation followed the rhythmic component of the LFP (according to the Rayleigh test). The firing of this neuron was significantly phase locked during sucrose processing but not during quinine processing, despite firing similar overall numbers of action potentials in response to each taste. Across the entire population $(n=109)$, 50 neurons fired in a phase-modulated way to at least one taste; of these, the majority $(n=32)$ were significantly phase locked for only one or two of the four tastes (Fig. 8D). Most neurons that were significantly modulated (i.e., $p<0.05$ ) by the underlying network rhythm for at least one taste were completely lacking in phase modulation (i.e., $p>0.6$ ) for at least one other taste.

There was no apparent pattern regarding which taste(s) coupled into the rhythm. Although more neurons were significantly phase modulated during sucrose administration $(n=31)$ than during the other three tastes (mean of $n=25$ ), the range was small and no significant differences were observed (Fig. $8 E$ ). Furthermore, the pattern of significant phase modulation was very different from the pattern of taste responsiveness itself (see below); single-neuron taste responses recorded in awake rats using multiple trials tend to be very broad (i.e., most neurons respond to three or four tastes) (Katz et al., 2001a; Stapleton et al., 2006). Still, these results were similar to those observed when only tasteresponsive neurons were analyzed, probably because many neurons identified as "nonresponsive" by classic methods may participate in correlative coding (Katz et al., 2002; Jones et al., 2007).

Although differences in firing rates can in theory drive differences in spike-to-wave correlation, they did not appear to do so here. The neuron in Figure $8 C$, for instance, responded similarly (in terms of spikes per second) to administration of both tastes (supplemental Fig. 2, available at www.jneurosci.org as supplemental material), but the response was coupled to the running rhythm only for sucrose. Across the entire sample, the average within-neuron correlation between the significance of modulation depth ( $p$ value) and firing rate was mildly negative (mean of $r=-0.12$ ), which suggests that firing rate had only a very small impact on the results (a neuron-by-neuron presentation of these data can be found in supplemental Fig. 3, available at www. jneurosci.org as supplemental material). Clearly, the taste specificity of neural assemblies was not an artifact of taste-specific single-neuron firing rates.

Finally, our data suggest that the early and late taste rhythms, although distinct in several ways, are parts of a single, evolving process. There were few neurons for which $7-12 \mathrm{~Hz}$ coupling was significantly different for early and late rhythms; most (Fig. $8 \mathrm{~F}$ ) (supplemental Fig. 4, available at www.jneurosci.org as supplemental material) were significantly modulated through boththat is, phase modulation lasted across a full $2 \mathrm{~s}$ of poststimulus time-although there appears to be a subtle change in the spiketo-LFP phase relationship [with spikes clustering just before the peak during the early taste rhythm and just after the peak during the late taste rhythm (supplemental Fig. 5, available at www. jneurosci.org as supplemental material)]. Although our analysis is limited by short time spans ( $1 \mathrm{~h}$ sessions before loss of attention) and few trials (only $\sim 10$ deliveries of each taste are possible in that hour), the lack of a trend toward neurons being modulated by only one or the other of the taste rhythms and not both is striking; stimulus-to-stimulus variability in modulation is higher by several orders of magnitude. We conclude that the same neurons are involved in the early and late taste rhythms.

\section{Discussion}

\section{Multiple 7-12 Hz rhythms characterize sensory cortical function}

Although the $7-12 \mathrm{~Hz}(\alpha$ or $\mu$ ) band in neural recordings is frequently discussed as if it were a single power peak with an ascribable function, our results, which provide the first report of this rhythm in GC, reveal three distinct 7-12 Hz rhythms in sensory cortex within single recording sessions. In the attentive/ aroused rat, stimulus delivery causes a large, short-latency gustatory evoked potential which "kicks off" a 10-11 Hz early taste rhythm. This rhythm then slows down such that, by $\sim 1 \mathrm{~s}$ after stimulus delivery, it has restabilized as a $7-8 \mathrm{~Hz}$ late taste rhythm. A third, higher-amplitude rhythm, centered on $\sim 9 \mathrm{~Hz}$, appears in spontaneous activity at $\sim 1 \mathrm{~h}$ into the session, a general inattentive rhythm that can be observed in a wide range of sensory cortices (Tiihonen et al., 1989; Pfurtscheller et al., 1997; Mölle et al., 2002; Bezdudnaya et al., 2006; Fontanini and Katz, 2006; Womelsdorf and Fries, 2007). This last rhythm is obviously not specific to taste processing [although it has been shown to drastically change single-neuron taste coding to maximize differences between palatable and aversive tastes (Fontanini and Katz, 2006)], but the early and late rhythms almost certainly are: they are induced by taste delivery, they are initially phase locked to taste delivery, the early-to-late shift occurs at the same time as the previously described shift in the content of single-neuron taste temporal codes (Fontanini et al., 2009), and they recruit tastespecific ensembles of phase-locked neurons.

In addition to differing in time of occurrence, peak frequency (early taste $>$ inattentive $>$ late taste), and amplitude (inattentive $\gg$ late taste $>$ early taste), the three rhythms differ from each other in wave shape (the early and late taste rhythms are approximately sinusoidal, whereas the inattentive rhythm is saw-toothed) and in their interrelationships: in the context of pre-taste (inattentive) rhythmicity, the peak frequency of the early taste rhythm is significantly reduced, whereas that of the late taste rhythm is unaffected. Although LFP data does not allow us to reach conclusions regarding underlying physiological mechanisms, our results clearly demonstrate that these three spectral peaks differ from each other in many ways and thus are likely non-identical at the cellular level (see below); furthermore, we can say that they appear de novo, are 
A

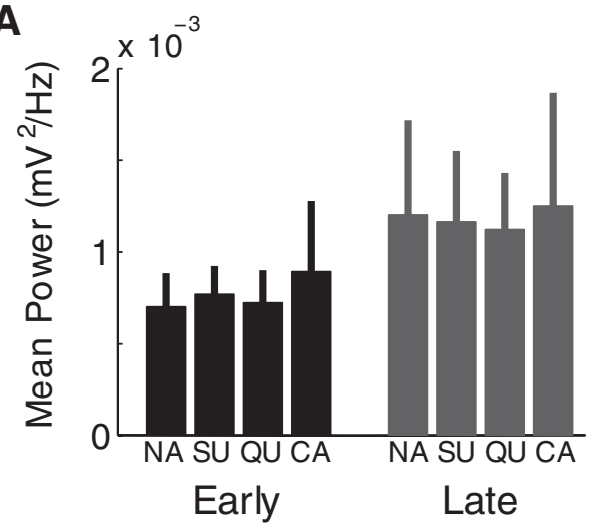

B

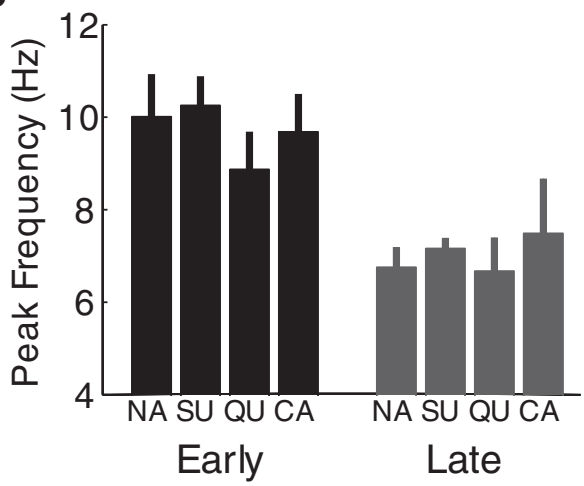

C
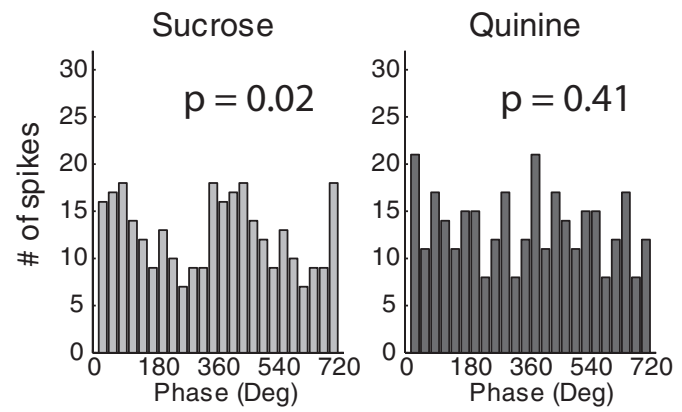

D

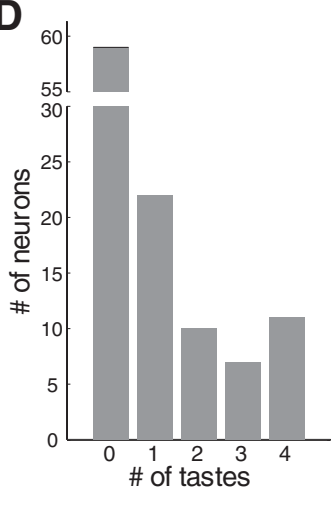

E
F

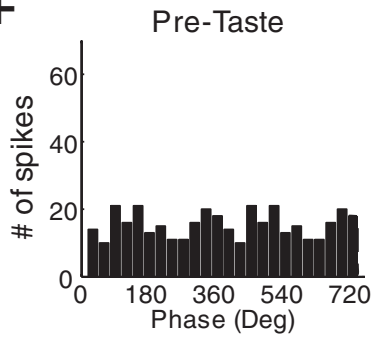

Early Post-Taste
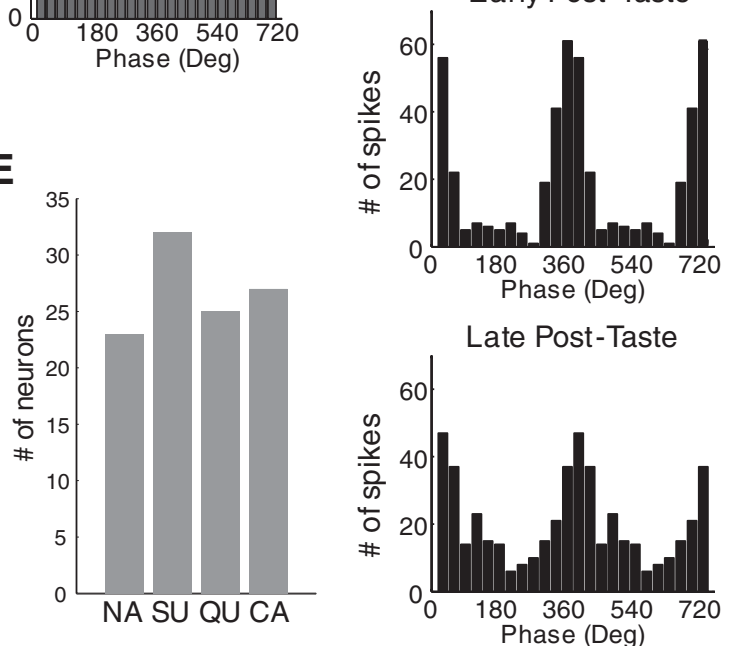

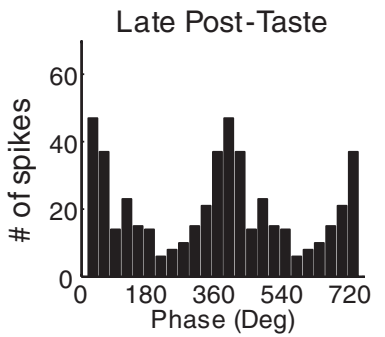

Figure 8. Taste specificity in 7-12 Hz synchrony. $\boldsymbol{A}$, The average 7-12 Hz power in GC LFPs did not reveal any significant taste specificity for either the early or late taste rhythms. $\boldsymbol{B}$, No significant taste specificity was apparent in the peak frequencies of either the early or late taste rhythms. C, A comparison of the number of action potentials fired by one $\mathrm{GC}$ neuron ( $y$-axis) to sucrose and quinine, keyed to phases of the 7-12 Hz cycle in the LFP ( $y$-axis). The neuron showed a biphasic firing rate response to both stimuli (supplemental Fig. 2, available at www.jneurosci.org as supplemental material), but this response was phase modulated, i.e., coupled to the $7-12 \mathrm{~Hz}$ rhythm, only in response to sucrose. $\boldsymbol{D}$, A frequency histogram showing the number of neurons ( $y$-axis) for which poststimulus responses were significantly phase modulated to $0,1,2,3$, or 4 of the administered stimuli. $\boldsymbol{E}$, A frequency histogram showing how often each taste caused significantly phase-modulated activity. $\boldsymbol{F}$, A representative example neuron, showing no phase-related firing in the prestimulus period (top), but strong phase-related firing in both early (middle) and late (bottom) portions of the sensory response. NA, Sodium chloride; SU sucrose; QU, quinine; CA, citric acid.

kick-started by a large GEP, and are maintained by network mechanisms (Figs. 2, 3).

The function of $7-12 \mathrm{~Hz}$ activity is a subject of much debate in the current literature. A number of researchers, noting that 7-12 $\mathrm{Hz}$ rhythms arise during periods of immobility or unresponsiveness, have suggested that $\alpha / \mu$ represents either physiological (Tiihonen et al., 1989; Pfurtscheller et al., 1997; Mölle et al., 2002; Bezdudnaya et al., 2006; Fontanini and Katz, 2006) or pathological (Steriade and Amzica, 2003; Shaw, 2007) "idling," a withdrawal from, and gating of, sensory input; we have suggested on the basis of our single-neuron taste data that this idling represents not simple loss of attention but a refocusing on broad palatability distinctions (Fontanini and Katz, 2006), an explanation that is consistent with this rhythm changing the taste rhythms. Other researchers have interpreted peaks in this frequency band as evidence of active inhibition: filtering out "top-down" signals from neocortex that might otherwise compete with processing of the attended stimuli during perceptual tasks (i.e., not just passive failure to process) (Kelly et al., 2006; Klimesch et al., 2007) or filtering out bottom-up inputs during memory tasks (Klimesch, 1999; Tuladhar et al., 2007; Payne and Kounios, 2009). In the context of this latter result, it has been suggested that $\alpha / \mu$ may represent a state of internal attention (Cooper et al., 2006).

The present results suggest a potential explanation for the variety of interpretations described above: different researchers may be reaching divergent conclusions concerning the function of $\alpha / \mu$ because they are actually recording distinctly different rhythms in their preparations. Just as pretaste rhythm appears only under certain conditions (when the rat has become "bored" with the experimental context), it is possible that one $7-12 \mathrm{~Hz}$ rhythm might dominate one researcher's experimental setup, whereas another dominates another's, particularly if the two are using different species and recording methods.

Even simple and subtle differences in the precise stimulus being delivered likely influence which rhythm is induced during sensory processing: our previous research on taste temporal codes suggests that stimulus identity coding occurs in the first second of the taste response, a period coinciding with early taste processing rhythm (i.e., the $10.5 \mathrm{~Hz}$ rhythm), whereas the late taste processing rhythm $(7 \mathrm{~Hz})$ coincides with a period of the taste response characterized by palatability coding, evaluation of the emotional properties of the taste (Katz et al., 2001a; Fontanini and Katz, 2006; Grossman et al., 2008). The specific frequency that dominates a sensory response may thus depend on the emotional salience of the stimuli used.

Regardless of which previously investigated characterizations-idling, active inhibition, internal attention, or memorial process-best fits one or another of our observed rhythms, our data suggest that one function of $7-12 \mathrm{~Hz}$ rhythmicity in GC might be to organize stimulus-specific cell assemblies, such that coincidence detectors could conceivably "read" the GC output signal and "decode" the taste delivered to the tongue (note that many other decoding strategies are likely in play as well; phaselocked firing is not the only reasonable metric of taste-related 
activity) (Katz et al., 2001, 2002). This is a somewhat surprising result, because the vast majority of research examining this issue has consistently implicated $\gamma$ rhythm-variously described as spanning the bandwidth between 30 and $80 \mathrm{~Hz}$ (Lisman, 2005; Fries et al., 2007; Kay et al., 2009)—as the rhythm responsible for binding cell assemblies together (Singer, 1990; Olufsen et al., 2003; Lisman, 2005; Womelsdorf et al., 2006). For taste-specific assemblies in GC, an analogous function may be played by rhythms in the 7-12 Hz range: perhaps in a cortex lacking the usual columnar structure, a cortex that furthermore is known to subtend a notoriously "slow" sensory system (Travers and Norgren, 1986; Katz et al., 2001a), 7-12 Hz rhythms perform the job that in other cortices is performed by $\gamma$.

\section{Multiple peak frequencies in one response}

The fact that the late taste rhythm tends to reliably follow the early taste rhythm, just as palatability-related processing follows identity coding and palatability-specific behaviors follow nondistinctive licking, suggests that the two rhythms might be related in function and/or genesis. One obvious possibility is that one or both taste rhythms primarily reflect the rats' motor activity, most notably licking. The timing of licking corresponds poorly to the timing of the late taste rhythm, however, in that rats lick strongly at 6-7 Hz during the height of the $10.5 \mathrm{~Hz}$ early taste rhythm (Travers and Norgren, 1986; Gutierrez et al., 2006) and produce mainly lower-frequency ingestive behaviors at the height of the 7 $\mathrm{Hz}$ late taste rhythm (Travers and Norgren, 1986; Dinardo and Travers, 1994; Venugopal et al., 2007).

Furthermore, our data suggest that the early and late taste rhythms may be somehow related at some level: single neurons stay coupled into taste-specific assemblies across the time at which peak frequency changes. Recent work has offered a mechanistic explanation for how a single network (in this case, thalamus) might undergo such a mid-response change in peak frequency. Thalamic slices were shown to produce oscillatory LFPs in a 9-11 $\mathrm{Hz}$ rhythm via perfusion with a metabotropic glutamate receptor 1a (mGluR1a) agonist. The frequency of these network oscillations could be reduced to the vicinity of $4-6 \mathrm{~Hz}$ very simply, by decreasing the concentration of the mGluRla perfusion (Hughes et al., 2004). This in vitro slowing resembles that observed here quite strikingly and is consistent with the suggestion that single sensory cortical networks can begin oscillating at one frequency and then suddenly shift to a lower frequency. It is entirely possible that our early and late taste rhythms, which differ in many ways, are nonetheless produced by slightly different settings of a single network.

It is worth noting, in this context, that the lower-frequency late taste rhythm may in fact dip below the $\alpha$ range and into the 4-7 Hz band typically referred to as $\theta$ rhythm (Raghavachari et al., 2001). A great deal of research supports the suggestion that the $\theta$ rhythm plays an important role in the process of effortful encoding of memories (Sederberg et al., 2003). Because single presentations of novel tastes are known to induce learning and learning-related plasticity (Berman and Dudai, 2001), it could be suggested that the shift from early to late taste rhythms accompanies a switch from mainly perceptual (identifying the taste) to mainly memorial (marking the taste as familiar) function. The fact that single neurons shift from stimulus-identity coding to coding of emotional (palatability) properties at this precise time point (Katz et al., 2001a; Fontanini and Katz, 2006; Grossman et al., 2008) is consistent with this possibility, because it is these latter properties that track stimulus familiarity.

\section{Conclusion}

Peak frequency is just one variable of many with which brain rhythms can be characterized. Context-specific cross-regional coherence can be highly informative as to the function of a rhythm (Bressler et al., 1993), as can coupling between 7-12 Hz and other, higher-frequency rhythms (Lakatos et al., 2007; Palva and Palva, 2007; Isler et al., 2008; Payne and Kounios, 2009). In fact, the functional significance of $7-12 \mathrm{~Hz}$ rhythms may be more closely related to this cross-frequency, interregional coupling than to the local amplitude and frequency of the rhythm itself. It can be argued, therefore, that it is overly simplistic to create monolithic characterizations of the function of rhythms in particular frequency bands. In this context, it is worth noting that our data suggest possible elevations in $30-60 \mathrm{~Hz}$ power cooccurring with the early taste rhythm and in $14-20 \mathrm{~Hz}$ power accompanying (and a possible harmonic of) the late taste rhythm (Fig. 1C).

Although this is speculative, our data confirm the merit of such speculation. Within a single stimulus-sampling session, and in fact within a single $3 \mathrm{~s}$ period (from $1 \mathrm{~s}$ before stimulus delivery to $2 \mathrm{~s}$ after stimulus delivery), gustatory cortical LFPs can progress through a sequence of three distinct rhythms, all confined to the 7-12 Hz range. Although it may be argued that this can be explained simply in terms of increased specificity of peak frequency (e.g., $10-11 \mathrm{~Hz}$ rhythm "does" one thing, 7-8 Hz rhythm "does" another), we suggest that it might be more profitable to take a more systems-oriented perspective. Gustatory cortex is embedded in a dynamical network (Jones et al., 2006), and, as such, the peak frequencies expressed in GC LFPs should vary as a function of (1) neurotransmitter levels, (2) where dominant inputs are coming from, and (3) oscillation frequencies in these other inputs. Our speculation is that our data might offer clues to an unfolding perceptual process, in which changes in rhythmicity reflect changes in the dominant direction of "information flow": top-down pretaste inhibition of GC (Min and Herrmann, 2007) gives way to ascending signals carrying sensory information to GC (during which time early taste rhythm is observed) and then to amygdala-cortical signals carrying necessary evaluative taste information to GC (Grossman et al., 2008; Fontanini et al., 2009). True sensory process is not so much a matter of representation as of constant transformation.

\section{References}

Baird JP, St John SJ, Nguyen EA (2005) Temporal and qualitative dynamics of conditioned taste aversion processing: combined generalization testing and licking microstructure analysis. Behav Neurosci 119:983-1003.

Bartoshuk LM, McBurney DH, Pfaffmann C (1964) Taste of sodium chloride solutions after adaptation to sodium chloride: implications for the “water taste." Science 143:967-968.

Berman DE, Dudai Y (2001) Memory extinction, learning anew, and learning the new: dissociations in the molecular machinery of learning in cortex. Science 291:2417-2419.

Bermejo R, Houben D, Zeigler HP (1998) Optoelectronic monitoring of individual whisker movements in rats. J Neurosci Methods 83:89-96.

Bezdudnaya T, Cano M, Bereshpolova Y, Stoelzel CR, Alonso JM, Swadlow HA (2006) Thalamic burst mode and inattention in the awake LGNd. Neuron 49:421-432.

Bollimunta A, Chen Y, Schroeder CE, Ding M (2008) Neuronal mechanisms of cortical alpha oscillations in awake-behaving macaques. J Neurosci 28:9976-9988.

Bressler SL, Coppola R, Nakamura R (1993) Episodic multiregional cortical coherence at multiple frequencies during visual task performance. Nature 366:153-156.

Bruns A (2004) Fourier-, Hilbert- and wavelet-based signal analysis: are they really different approaches? J Neurosci Methods 137:321-332.

Cooper NR, Burgess AP, Croft RJ, Gruzelier JH (2006) Investigating evoked and induced electroencephalogram activity in task-related alpha power 
increases during an internally directed attention task. Neuroreport 17:205-208.

Delorme A, Makeig S (2004) EEGLAB: an open source toolbox for analysis of single-trial EEG dynamics including independent component analysis. J Neurosci Methods 134:9-21.

Dinardo LA, Travers JB (1994) Hypoglossal neural activity during ingestion and rejection in the awake rat. J Neurophysiol 72:1181-1191.

Duffy FH, Albert MS, McAnulty G, Garvey AJ (1984) Age-related differences in brain electrical activity of healthy subjects. Ann Neurol 16:430-438.

Fanselow EE, Sameshima K, Baccala LA, Nicolelis MA (2001) Thalamic bursting in rats during different awake behavioral states. Proc Natl Acad Sci U S A 98:15330-15335.

Fontanini A, Katz DB (2005) 7 to $12 \mathrm{~Hz}$ activity in rat gustatory cortex reflects disengagement from a fluid self-administration task. J Neurophysiol 93:2832-2840.

Fontanini A, Katz DB (2006) State-dependent modulation of time-varying gustatory responses. J Neurophysiol 96:3183-3193.

Fontanini A, Grossman SE, Figueroa JA, Katz DB (2009) Distinct subtypes of basolateral amygdala taste neurons reflect palatability and reward. J Neurosci 29:2486-2495.

Freunberger R, Klimesch W, Griesmayr B, Sauseng P, Gruber W (2008) Alpha phase coupling reflects object recognition. Neuroimage 42:928-935.

Fries P, Nikolić D, Singer W (2007) The gamma cycle. Trends Neurosci 30:309-316.

Grossman SE, Fontanini A, Wieskopf JS, Katz DB (2008) Learning-related plasticity of temporal coding in simultaneously recorded amygdala-cortical ensembles. J Neurosci 28:2864-2873.

Gutierrez R, Carmena JM, Nicolelis MA, Simon SA (2006) Orbitofrontal ensemble activity monitors licking and distinguishes among natural rewards. J Neurophysiol 95:119-133.

Hughes SW, Lörincz M, Cope DW, Blethyn KL, Kékesi KA, Parri HR, Juhász G, Crunelli V (2004) Synchronized oscillations at alpha and theta frequencies in the lateral geniculate nucleus. Neuron 42:253-268.

Isler JR, Grieve PG, Czernochowski D, Stark RI, Friedman D (2008) Crossfrequency phase coupling of brain rhythms during the orienting response. Brain Res 1232:163-172.

Jones LM, Fontanini A, Katz DB (2006) Gustatory processing: a dynamic systems approach. Curr Opin Neurobiol 16:420-428.

Jones LM, Fontanini A, Sadacca BF, Miller P, Katz DB (2007) Natural stimuli evoke dynamic sequences of states in sensory cortical ensembles. Proc Natl Acad Sci U S A 104:18772-18777.

Katz DB, Simon SA, Nicolelis MA (2001a) Dynamic and multimodal responses of gustatory cortical neurons in awake rats. J Neurosci 21:4478-4489.

Katz DB, Simon SA, Nicolelis MAL (2001b) Electrophysiological studies of gustation in awake rats. In: Methods and frontiers in the chemical senses (Simon SA, Nicolelis MAL, eds), pp 339-357. Boca Raton, FL: CRC.

Katz DB, Simon SA, Nicolelis MA (2002) Taste-specific neuronal ensembles in the gustatory cortex of awake rats. J Neurosci 22:1850-1857.

Kay LM, Beshel J, Brea J, Martin C, Rojas-Líbano D, Kopell N (2009) Olfactory oscillations: the what, how and what for. Trends Neurosci 32:207-214.

Kelly SP, Lalor EC, Reilly RB, Foxe JJ (2006) Increases in alpha oscillatory power reflect an active retinotopic mechanism for distracter suppression during sustained visuospatial attention. J Neurophysiol 95:3844-3851.

Klimesch W (1999) EEG alpha and theta oscillations reflect cognitive and memory performance: a review and analysis. Brain Res Brain Res Rev 29:169-195.

Klimesch W, Sauseng P, Hanslmayr S (2007) EEG alpha oscillations: the inhibition-timing hypothesis. Brain Res Rev 53:63-88.

Könönen M, Partanen JV (1993) Blocking of EEG alpha activity during visual performance in healthy adults. A quantitative study. Electroencephalogr Clin Neurophysiol 87:164-166.

Lakatos P, Chen CM, O'Connell MN, Mills A, Schroeder CE (2007) Neuronal oscillations and multisensory interaction in primary auditory cortex. Neuron 53:279-292.

Lisman J (2005) The theta/gamma discrete phase code occuring during the hippocampal phase precession may be a more general brain coding scheme. Hippocampus 15:913-922.

Min BK, Herrmann CS (2007) Prestimulus EEG alpha activity reflects prestimulus top-down processing. Neurosci Lett 422:131-135.

Mölle M, Marshall L, Fehm HL, Born J (2002) EEG theta synchronization conjoined with alpha desynchronization indicate intentional encoding. Eur J Neurosci 15:923-928.

Nikouline VV, Wikström H, Linkenkaer-Hansen K, Kesäniemi M, Ilmoniemi RJ, Huttunen J (2000) Somatosensory evoked magnetic fields: relation to pre-stimulus mu rhythm. Clin Neurophysiol 111:1227-1233.

Norgren R (1990) Gustatory system. In: The human nervous system (Paxinos G, ed), pp 845-861. New York: Academic.

Olufsen MS, Whittington MA, Camperi M, Kopell N (2003) New roles for the gamma rhythm: population tuning and preprocessing for the beta rhythm. J Comput Neurosci 14:33-54.

Palva S, Palva JM (2007) New vistas for alpha-frequency band oscillations. Trends Neurosci 30:150-158.

Payne L, Kounios J (2009) Coherent oscillatory networks supporting shortterm memory retention. Brain Res 1247:126-132.

Pfurtscheller G, Neuper C, Andrew C, Edlinger G (1997) Foot and hand area mu rhythms. Int J Psychophysiol 26:121-135.

Phillips MI, Norgren R (1970) A rapid method for permanent implantation of an intraoral fistula in rats. Behav Res Methods Instrum 2:124.

Raghavachari S, Kahana MJ, Rizzuto DS, Caplan JB, Kirschen MP, Bourgeois B, Madsen JR, Lisman JE (2001) Gating of human theta oscillations by a working memory task. J Neurosci 21:3175-3183.

Rojas-Líbano D, Kay LM (2008) Olfactory system gamma oscillations: the physiological dissection of a cognitive neural system. Cogn Neurodyn 2:179-194.

Sauseng P, Klimesch W, Gruber W, Doppelmayr M, Stadler W, Schabus M (2002) The interplay between theta and alpha oscillations in the human electroencephalogram reflects the transfer of information between memory systems. Neurosci Lett 324:121-124.

Sederberg PB, Kahana MJ, Howard MW, Donner EJ, Madsen JR (2003) Theta and gamma oscillations during encoding predict subsequent recall. J Neurosci 23:10809-10814.

Shaw FZ (2007) 7-12 Hz high-voltage rhythmic spike discharges in rats evaluated by antiepileptic drugs and flicker stimulation. J Neurophysiol 97:238-247.

Shumway RH, Stoffer DS (2005) Time series analysis and its applications. New York: Springer.

Singer W (1990) The formation of cooperative cell assemblies in the visual cortex. J Exp Biol 153:177-197.

Smith DV, St John SJ (1999) Neural coding of gustatory information. Curr Opin Neurobiol 9:427-435.

Stapleton JR, Lavine ML, Wolpert RL, Nicolelis MA, Simon SA (2006) Rapid taste responses in the gustatory cortex during licking. J Neurosci 26:4126-4138.

Steriade M, Amzica F (2003) Sleep oscillations developing into seizures in corticothalamic systems. Epilepsia 44 [Suppl 12]:9-20.

Tiihonen J, Kajola M, Hari R (1989) Magnetic mu rhythm in man. Neuroscience 32:793-800.

Tort AB, Kramer MA, Thorn C, Gibson DJ, Kubota Y, Graybiel AM, Kopell NJ (2008) Dynamic cross-frequency couplings of local field potential oscillations in rat striatum and hippocampus during performance of a T-maze task. Proc Natl Acad Sci U S A 105:20517-20522.

Travers JB, Norgren R (1986) Electromyographic analysis of the ingestion and rejection of sapid stimuli in the rat. Behav Neurosci 100:544-555.

Tuladhar AM, ter Huurne N, Schoffelen JM, Maris E, Oostenveld R, Jensen O (2007) Parieto-occipital sources account for the increase in alpha activity with working memory load. Hum Brain Mapp 28:785-792.

van der Meer MA, Redish AD (2009) Low and high gamma oscillations in rat ventral striatum have distinct relationships to behavior, reward, and spiking activity on a learned spatial decision task. Front Integr Neurosci 3:9.

Venugopal S, Travers JB, Terman DH (2007) A computational model for motor pattern switching between taste-induced ingestion and rejection oromotor behaviors. J Comput Neurosci 22:223-238.

Verhagen JV (2007) The neurocognitive bases of human multimodal food perception: consciousness. Brain Res Rev 53:271-286.

Welsh JP, Lang EJ, Suglhara I, Llinás R (1995) Dynamic organization of motor control within the olivocerebellar system. Nature 374:453-457.

Womelsdorf T, Fries P (2007) The role of neuronal synchronization in selective attention. Curr Opin Neurobiol 17:154-160.

Womelsdorf T, Fries P, Mitra PP, Desimone R (2006) Gamma-band synchronization in visual cortex predicts speed of change detection. Nature 439:733-736. 